\title{
Conception d'une séquence d'introduction dynamique du produit scalaire via une approche constructiviste intégrant la mécanique et les TIC
}

\section{Conception of a dynamic introduction sequence of the scalar product via a constructivist approach integrating mechanics and ICT}

DOI: $10.46932 / \mathrm{sfjdv} 2 \mathrm{n} 2-148$

Received in: March 1st, 2021

Accepted in: May 30th, 2021

\begin{abstract}
Khadija Raouf
Groupe de recherche interdisciplinaire sur les Systèmes d'Enseignement Apprentissage des Sciences et Technologies (SEAST), Domicilié au Centre Régional des Métier de l'éducation et de la formation (CRMEF), Casablanca-Settat, Section El Jadida, Avenue Brahim Roudani, 24000, El Jadida, Maroc Professeur habilité en didactique des sciences et formatrice des futurs enseignants des sciences physiques au CRMEF d'El Jadida, Maroc.

Email : raouf.crmef@gmail.com
\end{abstract}

\section{Najia Benkenza}

Groupe de recherche interdisciplinaire sur les Systèmes d'Enseignement Apprentissage des Sciences et Technologies (SEAST), Domicilié au Centre Régional des Métier de l'éducation et de la formation (CRMEF), Casablanca-Settat, Section El Jadida, Avenue Brahim Roudani, 24000, El Jadida, Maroc Professeur assistant et formatrice des futurs enseignants des mathématiques au CRMEF d'El Jadida, Maroc.

Email : benkenzanajat@gmail.com

\section{M'hamed El aydi}

Groupe de recherche interdisciplinaire sur les Systèmes d'Enseignement Apprentissage des Sciences et Technologies (SEAST), Domicilié au Centre Régional des Métier de l'éducation et de la formation (CRMEF), Casablanca-Settat, Section El Jadida, Avenue Brahim Roudani, 24000, El Jadida, Maroc Professeur assistant et formateur des futurs enseignants des mathématiques au CRMEF d'El Jadida,

Maroc.

Email: elaydi58@gmail.com

Mohamed Anaya

Groupe de recherche interdisciplinaire sur les Systèmes d'Enseignement Apprentissage des Sciences et Technologies (SEAST), Domicilié au Centre Régional des Métier de l'éducation et de la formation (CRMEF), Casablanca-Settat, Section El Jadida, Avenue Brahim Roudani, 24000, El Jadida, Maroc Inspecteur pédagogique des mathématiques, Direction provinciale d'El Jadida.

E-mail : anaya_mohamed@yahoo.fr

\section{khalid Ennaciri}

Groupe de recherche interdisciplinaire sur les Systèmes d'Enseignement Apprentissage des Sciences et Technologies (SEAST), Domicilié au Centre Régional des Métier de l'éducation et de la formation (CRMEF), Casablanca-Settat, Section El Jadida, Avenue Brahim Roudani, 24000, El Jadida, Maroc Enseignant du secondaire qualifiant, formateur retraité des futurs enseignants des mathématiques au

CRMEF d'El Jadida, Maroc.

E-mail: naciri2@gmail.com 


\title{
RÉSUMÉ
}

Le travail que nous présentons dans cet article vise la redynamisation de l'enseignement-apprentissage du produit scalaire via la conception d'une séquence d'introduction de cette notion au lycée et des modalités de sa mise en œuvre par l'enseignant. Cet intérêt est justifié par le manque d'activités motivantes dans les manuels marocains que nous avons consultés et par le fait que la construction de cette notion mobilise des objets mathématiques susceptibles de cumuler des difficultés d'apprentissages telles que les notions de vecteur et de produit. La démarche adoptée dans cette recherche, se base sur le constructivisme comme théorie d'apprentissage, l'approche interdisciplinaire pour donner plus de sens aux savoirs construits et l'intégration de la géométrie dynamique permettant de conjecture du résultat dans sa généralité en couvrant tous les cas de figures possibles et la construction éventuelle des figures dynamiques par les apprenants. Ainsi ; nous avons choisi comme activité d'approche une situation authentique dont la résolution fait appel essentiellement à la notion de résultante de deux forces que les apprenants ont déjà abordée en mécanique, et aux notions de somme de deux vecteurs et le théorème de Pythagore vus en mathématiques.

\begin{abstract}
The study presented in this article aims to revitalise the teaching and learning of the scalar product by conceiving a sequence for introducing this notion in high school and the modalities of its implementation by the teacher. This interest is justified by the lack of motivating activities in the Moroccan textbooks that we consulted and by the fact that the construction of this notion mobilises mathematical objects susceptible to cumulate learning difficulties such as the notions of vector and product. The approach adopted in this research is based on constructivism as a learning theory, the interdisciplinary approach to give more meaning to the knowledge built and the integration of dynamic geometry allowing to conjecture the result in its generality by covering all the possible cases of figures and the eventual construction of dynamic figures by pupils. Thus, we have chosen as an approach activity an authentic situation whose resolution calls essentially on the notion of resultant of two forces that the learners have already approached in mechanics, and on the notions of sum of two vectors and the Pythagorean theorem seen in mathematics.
\end{abstract}

\section{Introduction}

Le produit scalaire occupe une position centrale dans la plupart des domaines des sciences et techniques en l'occurrence, le domaine de l'électromagnétisme, l'hydrodynamique et la mécanique quantique. Dans le contexte scolaire, cette notion est utile pour la résolution de problèmes géométriques mobilisant distance, orthogonalité et angles et pour la vérification de l'orthogonalité de deux droites ou d'une droite et d'un plan. Au Maroc, le produit scalaire est abordé pour la première fois au tronc commun ( $1^{\text {ère }}$ année du lycée). Il est mobilisé en physique dans la modélisation de grandeurs physiques telles que le travail d'une force que les apprenants découvrent en $1^{\text {ère }}$ année Baccalauréat (2 $2^{\text {ème }}$ année du lycée).

Dans cette étude, le choix du produit scalaire, est motivé d'une part, par la pénurie ou la quasiabsence de recherche sur l'introduction de cette notion au niveau scolaire et d'autre part, par souci de dynamiser l'enseignement-apprentissage des mathématiques surtout que les démarches proposées dans la majorité des manuels du Tronc commun scientifiques que nous avons consultés, semblent limiter les 
fonctions des apprenants au statut d'exécutants de calculs ou de techniques sans opportunités de construction de sens.

Dans cette perspective et en harmonie avec les directives pédagogiques marocaines [1] et les programmes spécifiques à l'enseignement de mathématiques au secondaire qualifiant qui recommandent la promotion de la démarche de résolution de problèmes, nous proposons ici une séquence d'introduction du produit scalaire, basée sur une situation-problème réaliste et motivante issue des sciences physiques et qui peut faire appel à la géométrie dynamique. En plus de la situation-problème ; nous proposons des éléments qui peuvent aider à sa gestion en classe ainsi que les résultats à institutionnaliser. Notre travail est orienté par un cadre conceptuel et une démarche méthodologique dont nous donnons des brefs aperçus après la présentation de la problématique.

\section{PROBLEMATIQUE}

Dans le contexte scolaire marocain, le produit scalaire est introduit pour la première fois au tronc commun ( $1^{\text {ère }}$ année du lycée) à travers deux expressions : «l'expression géométrique » utilisant la projection orthogonale de l'un des deux vecteurs sur la direction de l'autre et «l'expression trigonométrique » utilisant les normes des deux vecteurs et le cosinus de leur angle. Certains manuels (et certains enseignants aussi) ajoutent une troisième expression avec les normes des deux vecteurs et la norme de leur somme. Nous considérons que la construction de cette notion mobilise, parmi d'autres, deux objets mathématiques susceptibles de cumuler des difficultés d'apprentissages ; qui sont les notions de "vecteur" et de "produit". En fait, la notion de "Vecteur" pose en elle seule de grandes difficultés à cause de la diversité des cadres dans lesquels elle est mobilisée (géométrique, algébrique, analytique, etc.).

A ceci s'ajoute des contraintes d'ordre sémiotique liées à la notion de "produit" qui a été souvent associée au symbole "x" et au fait que l'apprenant est habitué à une loi de composition interne alors que le résultat du produit scalaire est régi par une forme bilinéaire symétrique définie positive. En effet, l'apprenant s'attend à ce que le produit de deux vecteurs soit un vecteur, il découvre que le produit scalaire de deux vecteurs est un nombre réel.

Les résultats de lecture de quatre manuels de l'élève agrées par le ministère de tutelle révèlent que la majorité de ces manuels semblent réduire la notion du produit scalaire en une formule qu'on parachute après une série de démonstrations démunie de sens et d'articulation. On note l'absence de situations motivante et de conjecture via la géométrie dynamique qui permettrait aux apprenants de visualiser les différents cas de figures proposés pour introduire le produit scalaire. Partant de ces constats, notre présente recherche tente de répondre à la question suivante : 
Comment introduire la notion du produit scalaire à partir d'une situation problème motivante dont la démarche de résolution impose aux apprenants de mobiliser leurs prérequis et leurs représentations dans une perspective d'appropriation du sens de cette notion ?

\section{CADRE THÉORIQUE}

Fort de constater que les connaissances mathématiques apprises par les élèves ne peuvent être réduites à une partie de l'ensemble des connaissances enseignées, une large communauté didactique conçoit l'apprentissage dans une perspective constructiviste [2] où l'objet mathématique se construit sur des connaissances antérieures de l'apprenant et en fonction d'elles, tout en le plaçant au centre des apprentissages. Autrement dit la construction des savoirs et leur sens ne peuvent se faire que si l'apprenant est actif cognitivement puisqu'il ne s'agit pas seulement d'une affaire de représentations mais aussi d'activité mentale complexe et réflexive [3]. D'un point de vue didactique, le courant constructiviste s'intéresse plutôt aux procédures d'enseignement permettant de construire ou reconstruire les savoirs par l'apprenant et qui par conséquent s'oppose au modèle transmissif [4]. Les mêmes auteurs stipulent que :

"[...]D'une manière ou d'une autre, il s'agit d'admettre que les connaissances « se construisent ». Que ce soit de façon progressive et continue, ou bien par ruptures, l'activité du sujet conduit aux remodelages et aux réorganisations qu'implique l'apprentissage."

Dans une même optique, Sarrazy [5] souligne que la manière de faire faire des mathématiques est plus importante que ce qui est montré, enseigné et retenu. Ainsi pour que les apprenants puissent être actifs cognitivement et construire des liens entre leurs connaissances antérieures et les nouvelles connaissances à construire, il est nécessaire qu'ils passent par des phases d'assimilation et d'accommodation [6]. A ce niveau d'activités d'adaptation, l'apprenant actant ne peut anticiper ou reconnaître la connaissance produite ou son rôle et par conséquent une étape d'institutionnalisation s'avère nécessaire pour passer d'une organisation chronogénétique didactique à une topogénétique scolaire officielle [7]. Pour se faire, l'intégration de situations signifiantes pour les apprenants est essentielle puisqu'elle va leur permettre, entre autres, la dialectique entre connaissances, savoirs-objets d'enseignement et exigences de la situation. En effet, Perrenoud [3] atteste que le sens se construit pour une part, en situation, bref sur le vif et dans une interaction qui le module puisque cette activité dialectique réflexive ne peut s'opérer en dehors de contexte favorisant l'interaction des connaissances du sujet avec l'environnement physique et social. D'ailleurs, cette dimension interactive et actionnelle est l'une des assises clé du constructivisme.

De telles situations devraient permettre l'enrôlement de l'apprenant dans le processus d'apprentissage, le développement de la motivation et l'engagement vis-à-vis des activités proposées et 
les savoirs constitués. Brousseau [8] fondateur de la théorie des situations, classifie les situations sous trois types constituants des modèles différents de la rencontre négociée entre un apprenant et une connaissance. Il s'agit de situation d'action, situation de formulation et situation de validation [9] que Brousseau [8] explicite comme suit :

\footnotetext{
" Une situation modélise les enjeux et les responsabilités de décision d'un actant dans un certain milieu. Elle est choisie de telle manière que la stratégie de résolution ne puisse être mise en œuvre que grâce à une certaine connaissance mathématique"
}

Dans ce contexte, l'étape de dévolution est un élément clé du contrat didactique où l'apprenant est invité à assumer le statut d'actant dans une situation où il est amené à apporter des solutions sans culpabilité en cas d'échec. Le statut de l'erreur devrait s'inscrire dans une logique qui considère que l'apprentissage est un processus historique et stochastique tel qu'il est décrit par Brousseau [7].

Dans la construction de sens des apprentissages mathématiques, les questions de guidance revêtent une grande importance en suscitant l'interaction entre l'apprenant, la situation, le savoir mathématique médiatisé et l'enseignant [10]. D'où l'intérêt de choix pertinents des questions structurantes visant le développement d'un raisonnement progressif et approfondie [11]. D'ailleurs, Elstgeest [12] qualifie les bonnes questions de questions "productive ".

\footnotetext{
" The right question leads to where the answer can be found: to the real objects or events under study, there where the solution lies hidden. The right question asks children to show rather than to say the answer: they can go and make sure for themselves. I would like to call such questions 'productive' questions, because they stimulate productive activity."
}

Dans cette perspective constructiviste voire socioconstructiviste et motivationnelle, il s'avère pertinent d'intégrer la dimension interdisciplinaire ainsi les notions échappent au cloisonnement des disciplines et les liens entre ces notions sont explicités à travers des situations et des familles de situations. De même, l'utilisation pertinente des TIC dans une logique d'enseignement-apprentissage et de pédagogie active ne peut être que bénéfique dans la limite où les supports utilisés offrent à l'apprenant, des opportunités d'interaction avec le savoir et permettent une modélisation préalable aux activités de démonstration ou des démarches de résolution de problème en mathématiques, entre autres. En effet, comme précisé par Garcia [13], l'utilisation des TIC offre un large éventail de possibilités pouvant être exploitées par les enseignants afin de favoriser le processus d'enseignement-apprentissage et développer l'autonomie chez l'apprenant.

L'un des environnements logiciels de construction géométrique que nous avons exploité dans les travaux de cette recherche est la géométrie dynamique et en l'occurrence le logiciel Geogebra. Cette intégration devrait permettre le renouvellement des mathématiques enseignés en évitant la présentation 
linéaire et en mettant en œuvre des situations intéressantes avec une économie de temps [14]. En effet, les outils de la géométrie dynamique aident à rendre les objets abstraits plus concrets puisqu'ils permettent aux apprenants de visualiser des objets mathématiques, de décrire ou de construire une figure donnée, de mettre en évidence des propriétés géométriques de façon dynamique, et d'émettre ou vérifier des conjectures [15]. Sur le plan des attitudes et de la motivation des apprenants, des travaux de recherche ont révélé une influence positive de l'utilisation des outils de la géométrie dynamique sur ces deux caractéristiques [16-17]. D'autres recherches ont révélé que leur intégration dans un environnement numérique structuré a contribué d'une façon significative à la dynamique des apprentissages et à la progression des performances des sujets, en termes de score [18-20]. D'autre part et selon les orientations pédagogiques marocaines relatives à l'enseignement des mathématiques au secondaire qualifiant [1], l'outil informatique dans les nouveaux curricula revêt une considération spéciale, en raison du rôle qu'il occupe dans divers domaines et en tenant compte des orientations et choix énoncés dans la charte nationale de l'éducation et de la formation concernant le champ d'utilisation des TICE. Cependant, une exploitation optimale de ces logiciels est atteinte à travers l'implication des apprenants dans le processus de construction et de découverte.

\section{ELÉMENTS MÉTHODOLOGIQUES}

Dans cette étude, nous avons d'abord procédé à la lecture de manuels de l'apprenant du tronc commun agrées par le ministère de tutelle, en particulier l'introduction du produit scalaire. Puis nous nous sommes intéressés aux orientations pédagogiques officielles spécifiques à l'enseignement des mathématiques. L'objectif étant de tenter de comprendre les choix faits par les concepteurs des manuels consultés en analysant leur degré d'alignement aux exigences et recommandations institutionnelles. Or la démarche adoptée dans la majorité de ces manuels semble être axée sur des activités désarticulées, issues dans leur globalité d'un domaine purement mathématique, trop guidées à notre sens et par conséquent ne laissent aucune place à l'imagination ou à la créativité. Les séries de questions ou d'instructions qui sont censées guider l'apprenant dans son processus de démonstration, sont généralement parachutées. On a noté aussi l'absence d'intégration des TIC ou de situation interdisciplinaire ou de situation problème permettant la motivation des apprenants à s'engager activement dans le processus de construction de leurs apprentissages et la construction de sens et d'utilité de cette nouvelle notion à savoir le produit scalaire. Parmi les recommandations officielles spécifiques au chapitre produit scalaire, il est précisé de se limiter, à ce niveau, aux expressions faisant intervenir la projection orthogonale, et celle trigonométrique tandis que l'expression analytique est considérée hors programme.

Après concertation avec des enseignants des mathématiques à propos des difficultés réelles qu'ils 
rencontrent dans l'introduction du produit scalaire au tronc commun scientifique, notre équipe pluridisciplinaire constituée d'un inspecteur de mathématiques, de professeurs, et de formateurs des futurs enseignants des mathématiques et de la physique, nous avons conçu une situation problème la plus authentique possible, dont la résolution mobilise, d'une façon intégrée, des connaissances déjà vues en mathématiques et en partie en physique et des propositions de gestion des activités au profit de l'enseignant qui pourrait les adapter en fonction des conditions de la situation d'enseignementapprentissage propre à sa classe.

Notre démarche se veut constructiviste, interdisciplinaire et intégrant la géométrie dynamique pour permettre aux élèves d'émettre les conjectures adéquates, et de réaliser que la mesure de l'angle entre les deux vecteurs est aussi importante que leurs normes dans le calcul de leur produit scalaire.

\section{RÉSULTATS ET DISCUSSION}

Eu égard de l'importance des situations déclenchantes dans la motivation des apprenants et la structuration des activités d'apprentissage, nous avons opté pour une situation simple issue de la mécanique que les apprenants ont déjà étudié à savoir la résultante de deux forces que nous avons formulé ainsi :

Pour aligner le grand navire «AMWAGES » sur le quai, on se sert, comme illustré dans la figure1, de deux bateaux remorqueurs $\mathrm{A}$ et $\mathrm{B}$ qui le tirent en exerçant dessus deux forces $\overrightarrow{\mathbf{F 1}}$ et $\overrightarrow{\mathbf{F} 2}$ d'intensités déterminées. Les deux remorqueurs se déplacent selon deux directions faisant entre elles un angle $\theta$. On veut remplacer les deux remorqueurs par un seul, et on se demande quelle est l'intensité de la force $\overrightarrow{\mathbf{F}}$ qu'il doit être capable d'exercer sur le navire?

Fig. 1. Illustration de la situation déclenchante

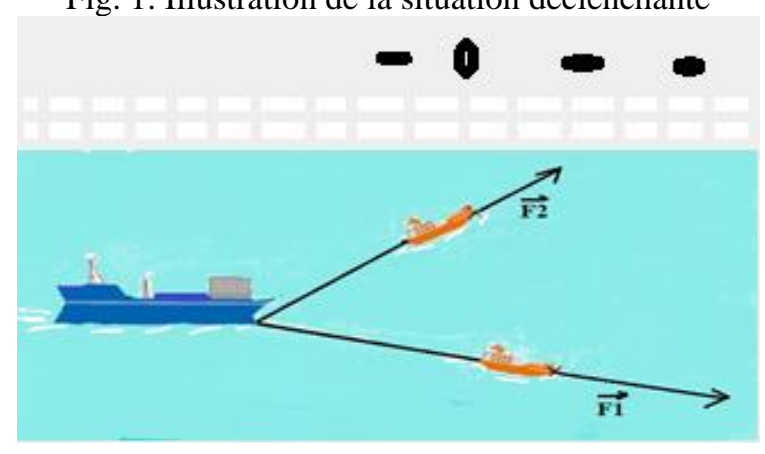

Cette situation problème interpelle dans sa résolution, des prérequis déjà traités en physique et en mathématiques : En mécanique, les apprenants du tronc commun ont déjà étudié au 1er trimestre, que la résultante est une force dont l'effet est équivalent à celui des forces appliquées à un corps. Cependant, l'objectif principal, est l'étude de l'équilibre statique d'un corps soumis à deux ou trois forces via la 
méthode graphique (polygone des forces) et la méthode analytique. Le choix de la résultante d'une force plutôt que la notion de travail d'une force, bien que cette dernière soit pertinente, est dû au fait que dans le contexte marocain, la notion du travail n'est plus abordée au tronc commun mais en $1^{\text {ère }}$ année Baccalauréat et par conséquent son adoption ne pourrait que cumuler les difficultés. En mathématiques, les apprenants ont déjà abordé des connaissances relatives à la détermination de la somme de deux vecteurs, le théorème de Pythagore, les propriétés des angles, la projection orthogonale et la trigonométrie. On note qu'il aurait été plus simple, à notre sens, d'introduire le produit scalaire via la méthode analytique que les apprenants utilisent déjà en mécanique pour établir la condition d'équilibre d'un solide soumis à trois forces à titre d'exemple. Ensuite, l'approche trigonométrique et l'approche géométrique pourraient être exploitées pour présenter les autres expressions du produit scalaire. Dans un souci de conformité avec les orientations pédagogiques officielles, cette option n'est pas prise en compte dans le modèle que nous proposons pour introduire le produit scalaire. Le choix de cette situation de physique comme activité d'approche permettrait le développement des capacités des apprenants à transférer leurs connaissances mathématiques dans le contexte d'une autre discipline et repérer d'une façon fluide les invariants structurels existants entre les deux situations comme illustré dans la figure 2 :

Fig. 2. Invariance structurelle entre la situation suggérée et celle des mathématiques


Cette démarche ne peut que favoriser le décloisonnement entre les disciplines d'une part et l'introduction du produit scalaire comme réponse à une question ainsi que l'initiation à d'autres utilités de cette notion, d'autre part.

Il est souhaitable dans la gestion de cette activité que l'enseignant, une fois la situation problème présentée et les consignes explicitées, envisage des mises en situation, d'abord de dévolution dont l'objectif étant de permettre aux apprenants de s'approprier la situation problème et de s'engager dans la recherche d'une solution à partir de leurs connaissances antérieures. Puis une situation d'action où l'apprenant mobilise ses connaissances antérieures pour développer une stratégie de résolution de la situation, une situation de formulation dans laquelle, il explicite la solution ou les solutions qu'il propose ainsi que le processus de résolution adopté et enfin une situation de validation où l'apprenant est invité à 
présenter son travail tout en argumentant ses choix et sa démarche de résolution de problème. L'institutionnalisation des savoirs incombe à l'enseignant qui en impliquant les apprenants procèdera au processus de généralisation en exploitant les autres cas de figure possibles explicités dans la figure 4 et 5 .

Cette approche de gestion de la situation-problème proposée s'inscrit dans une perspective constructiviste qui permettrait à l'apprenant de passer du statut de récepteur au statut d'actant, posture qui ne peut être que stimulante et le motiverait davantage à s'impliquer dans le processus d'apprentissage.

La modélisation et la mathématisation du problème comme illustré dans la figure3, permettra aux apprenants de réaliser que la résolution du problème revient à déterminer la longueur $\mathrm{AD}$ à partir de données connues telles que $\mathrm{AB}$ et $\mathrm{AC}$ et la mesure de l'angle $\alpha$.

Fig. 3. Modélisation et mathématisation du problème

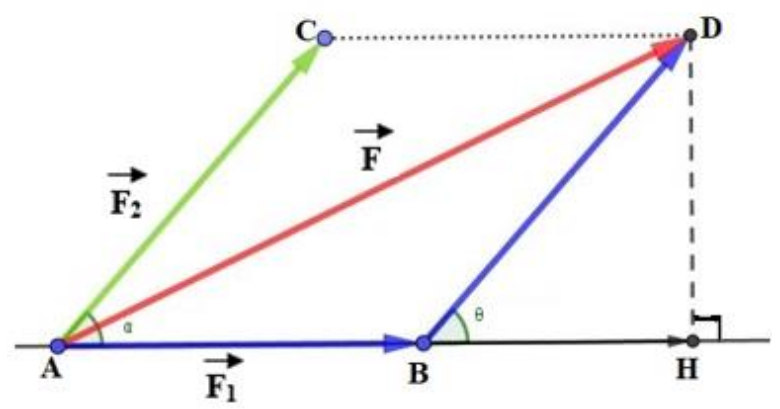

Il s'agit pour les apprenants de mobiliser entre autres, les prérequis relatifs à la projection orthogonale, l'égalité des angles, les propriétés du parallélogramme, les lignes trigonométriques et le théorème de Pythagore pour établir l'égalité :

$$
\mathrm{AD}^{2}=\mathrm{AB}^{2}+\mathrm{AC}^{2}+2 \mathrm{AB} \mathrm{AC} \cos (\alpha)
$$

Cette résolution mathématique permet la détermination de la longueur du vecteur $\overrightarrow{\mathbf{A D}}$ et par la suite, l'intensité $\mathrm{F}$ de la force $\overrightarrow{\mathbf{F}}$ résultante de $\overrightarrow{\mathbf{F 1}}$ et $\overrightarrow{\mathbf{F} 2}$, objectif à ne pas oublier pour donner du sens à l'activité proposée en particulier le pourquoi de la situation déclenchante. L'enseignant, dans son processus de construction des apprentissages, attirerait l'attention des apprenants sur le fait que l'intensité de cette force ne dépend que des intensités de $\overrightarrow{\mathbf{F 1}}$ et $\overrightarrow{\mathbf{F} 2}$ et de la mesure de l'angle formé par ces deux forces. En pratique, on remarque qu'en exerçant deux forces $\overrightarrow{\mathbf{F 1}}$ et $\overrightarrow{\mathbf{F} 2}$ d'intensités données sur un corps solide, on peut modifier l'intensité de leur résultante rien qu'en modifiant la mesure de l'angle entre ces deux forces ce qui illustre l'importance de l'expression : $2 \mathrm{~F}_{1} \mathrm{~F}_{2} \cos (\alpha)$. 
Selon le niveau des élèves et des conditions qui sont propres à chaque enseignant et à chaque groupe classe, l'enseignant pourrait exploiter ou pas les questions de guidance suivantes :

On représente :

- La force $\overrightarrow{\mathbf{F 1}}$ par le vecteur $\overrightarrow{\mathbf{A B}}$

- La force $\overrightarrow{\mathbf{F 2}}$ par le vecteur $\overrightarrow{\mathbf{A C}}$

- La force $\overrightarrow{\mathbf{F}}$ par le vecteur $\overrightarrow{\mathbf{A D}}$

On considère le point $\mathrm{H}$, projection orthogonale de $\mathrm{D}$ sur la droite $(\mathrm{AB})$

1) Quelle est la nature du triangle AHD ? en déduire que :

$$
\mathrm{AD}^{2}=\mathrm{AB}^{2}+\mathrm{BH}^{2}+2 \mathrm{AB} \mathrm{BH}+\mathrm{DH}^{2}
$$

2) Quelle est la nature du triangle BHD ? En déduire que :

$$
\mathrm{AD}^{2}=\mathrm{AB}^{2}+\mathrm{BD}^{2}+2 \mathrm{AB} \mathrm{BH}
$$

3) Exprimer $\cos (\theta)$ dans le triangle $\mathrm{BDH}$ et déduire que :

$$
\mathrm{AD}^{2}=\mathrm{AB}^{2}+\mathrm{BD}^{2}+2 \mathrm{AB} \mathrm{BD} \cos (\theta)
$$

Qu'on peut écrire aussi :

$$
\mathrm{AD}^{2}=\mathrm{AB}^{2}+\mathrm{AC}^{2}+2 \mathrm{AB} \mathrm{AC} \cos (\alpha)
$$

Il est important de songer à revenir à la situation déclenchante afin que les apprenants déduisent l'intensité de la force résultante des deux forces exercées par les deux bateaux remorqueurs à partir de l'équation 1. Il serait judicieux à ce niveau que l'enseignant attire leur attention sur les variables dont dépend l'intensité de cette force.

La démarche de résolution basée sur des questions de guidance, appuyée par une pratique enseignante délégant les tâches aux apprenants, vise, au besoin, le soutien de ces derniers dans la phase d'adaptation de leurs connaissances antérieures en vue de répondre aux questions posées. Ce mode d'action donnerait plus de sens aux situations que le simple traitement d'information. Pour amorcer l'étape de généralisation, à partir de laquelle l'annonce de la notion de produit scalaire sera faite, nous proposons l'intégration de la géométrie dynamique compte tenu de ses potentialités afin de visualiser le devenir de l'égalitél dans les autres cas de figures possibles suivants : 
Fig. 4. Cas où H est sur la demi-droite $(\mathrm{AB}]$ privée du segment $[\mathrm{AB}]$



Fig. 5. Cas où H est sur le segment $[\mathrm{AB}]$



Grâce à cet outil, les apprenants vont visualiser, avant de procéder aux activités de démonstration de l'égalité entre $\mathrm{AD}^{2}$ et $\mathrm{AB}^{2}+\mathrm{AC}^{2}+2 \mathrm{AB} \mathrm{AC} \cos (\alpha)$ et ce indépendamment de la longueur des vecteurs et la mesure de l'angle qu'ils forment entre eux. L'apprenant sera plus motivé s'il manipule lui-même le logiciel.

Après ce stade, on pourra passer à l'étape de généralisation de ces résultats où l'apprenant découvre la notion du produit scalaire via l'expression trigonométrique et saisi son utilité et le pourquoi de la situation déclenchante. En attirant l'attention de l'apprenant sur l'expression “ $\mathrm{AB} \mathrm{AC} \cos (\alpha)$ figurant dans l'égalité $\mathrm{AD}^{2}=\mathrm{AB}^{2}+\mathrm{AC}^{2}+2 \mathrm{AB} \mathrm{AC} \cos (\alpha)$, on introduit la notion du produit scalaire des deux vecteurs qu'on note $: \overrightarrow{\mathbf{A B}} \cdot \overrightarrow{\mathbf{A C}}$

Nous estimons, comme illustré par le modèle d'ingénierie que nous proposons, qu'il n'est pas nécessaire d'introduire, à ce stade, plus d'une expression du produit scalaire pour des raisons pédagogiques et de logique de structuration. Cette démarche permettrait aux apprenants de mieux maitriser le raisonnement mathématique et la démarche didactique préconisée.

\section{CONCLUSION ET PERSPECTIVES}

Considérant que pour introduire une nouvelle notion, aussi abstraite que le produit scalaire, il est utile de se baser sur des situation-problèmes pour aider les élèves à mobiliser leurs connaissances antérieures afin de les réorganiser de manière plus efficace. Et pour donner du sens à la nouvelle notion visée, nous estimons que la situation doit être riche, authentique et motivante; dans le sens qu'elle 
mobilise suffisamment de connaissances nécessaires pour définir la nouvelle notion et qu'elle pose un problème pertinent dans un contexte assez familier aux élèves.

Nous avons proposé une situation issue de la physique avec un contexte proche des élèves (El Jadida abrite l'un des grands ports du Maroc), pour souligner l'importance de l'interdisciplinarité dans la construction du sens en mathématique et pour inciter les élèves à interagir avec le problème posé et non avec les intentions didactiques de l'enseignant. Pour la gestion de l'activité avec les apprenants, nous proposons d'utiliser un logiciel de géométrie dynamique permettant de conjecturer l'effet sur l'intensité de la résultante de la norme de chacun des vecteurs et aussi de la mesure de l'angle entre eux ; ce qui va justifier l'importance de l'expression appelée " produit scalaire" par la suite.

Sachant que la meilleure façon pour valider ce genre de proposition est son expérimentation avec des élèves en classe, nous regrettons de ne pas avoir pu le faire dans ces conditions de la pandémie du Covid19. Mais nous tenons à le faire dès qu'il sera possible et y rendre compte dans un autre article. 


\section{RÉFÉRENCES BIBLIOGRAPHIQUES}

1. Orientations pédagogiques et curricula pour l'enseignement des mathématiques dans le cycle du secondaire qualifiant, Royaume du Maroc, Ministère de l'éducation nationale, de l'enseignement supérieur et de la recherche, (2007).

2. C. Laborde, Deux usages complémentaires de la dimension sociale dans les situations d'apprentissages en mathématiques. Dans Garnier, C. N. Bednarz, et I. Ulanovskaya, Après Vygotski et Piaget. Perspectives Sociale et Constructiviste. Ecole russe et occidentale. Pédagogies en développement. $3^{\mathrm{e}}$ édition, Bruxelles : De boeck, (2009).

3. P. Perrenoud, Métier d'élève et sens du travail scolaire, Collection Pédagogies, 6e édition Paris : ESF éditeur, (2005).

4. J.P. Astolfi, E. Darot et Y. Ginsburger-Vogel, Mots-clés de la didactique des sciences, repères, définitions, bibliographies. Pratiques pédagogiques. $2^{\mathrm{e}}$ édition. Bruxelles : De boeck, (2008).

5. B. Sarrazy, Ostension et dévolution dans l'enseignement des mathématiques. Éducation et didactique, 1(3), 31-46, (2007).

6. D. Masciotra, Le constructivisme en termes simples, Vie pédagogique, 143, 48-52, (2007).

7. G. Brousseau, Des dispositifs piagétiens...aux situations didactiques. Education et didactique, 6 (2), 103-129, (2012).

8. G. Brousseau, Glossaire de quelques concepts de la théorie des situations didactiques en mathématiques, (2003), [En ligne]. Disponible: http://perso.orange.fr/daest/guybrousseau/textes/glossaire_Brousseau.pdf

9. C. Cohen-Azria, B. Daunay, I. Delcambre, et D, Lahaner-Reuter, Dictionnaire des concepts fondamentaux des didactiques, $3^{\mathrm{e}}$ édition, Louvain-la-Neuve : De boek supérieur, (2013).

10. P. Jonnaert, Apprentissages des mathématiques en situation : une perspective constructiviste, R. S. E, 22(2), 233-252, (1996).

11. W. Harlen, Evaluation et pédagogie d'investigation dans l'enseignement scientifique : De la politique á la pratique, IAP Global Network of Science Academies. Édition française présentée par Edith Saltiel et Pierre Léna, (2013). [En ligne]. Disponible : https://www.interacademies.org/sites/default/files/publication/assessment_guide_french.pdf

12. J. Elsteeg, The right question at the right time. In Wynne Harlen. Primary Science: Taking the Plunge. Oxford, England : Heinemann Educational, pp. 36-46, (1985).

13. V. M. García, M.del. Guirao Carpes, J. M. Rabal Alonso, Teacher's perception about the importance of the use of ICT in the classroom. ITICOMTAS and ICOMTISM validation, South Florida Journal of Development, Miami, 2(2), 2287-2301, (2021).

14. M. Oudrhiri, De l'usage pédagogique du numérique dans l'enseignement des mathématiques au Maroc, Revue EPI. (2016). [En ligne]. Disponible : https://www.epi.asso.fr/revue/articles/al605c.htm 
15. H.E. Rajaonarimanana, et A. Totohasina, Initiation aux TIC par Geogebra dans des classes de mathématiques, Revue Didaktika, 3, 78-99, (2019).

16. R. Masri, T.S. Hiong, N.M. Tajudin, et Z. Zamzamir, The effects of using GeoGebra teaching strategy in Malaysian secondary schools: A case study from Sibu, Sarawak, Malaysian, J. Soc. Space, 12(7), 13-25, (2016).

17. A.B. Kamariah, M.A. Ahmad Fauzi, S.L. Wong, A.T. Rohani, Exploring secondary school students' motivation using technologies in teaching and learning mathematics, Procedia. Soc. Behav Sci, 2, 4650-4654, (2010).

18. N. Arbain et N.A. Shukor, The effects of Geogebra on student's achievement, Procedia. Soc. Behav Sci, 172, 208-214, (2015).

19. Abdoul Massalabi Nouhou, Impact de l'utilisation de GeoGebra sur l'apprentissage des élèves : Cas de la compréhension du concept de fonctions numériques au lycée, Education. Thèse de doctorat en Sciences de l'éducation, Université de Cergy Pontoise, France, (2019).

20. O. F. Tutkun, et B. Ozturk, The effect of Geogebra mathematical software to the academic success and the level of Van Hiele geometrical thinking, Int. J. Acad. Res, Part B, 5(4), 22-28, (2013). 